\title{
A Curious Case of Chronic Eosinophilic Pneumonia
}

\author{
Muhammad Zeeshan Siddiqui ${ }^{1, *}$, Taha Jawaid Khan ${ }^{2}$ and Samiya Khan ${ }^{3}$ \\ 1 Department Of Internal Medicine, Nassau University Medical Center, East Meadow, New York, NY \\ 11554, USA \\ 2 Department Of Internal Medicine, University of Oklahoma Health Sciences Center, Oklahoma City, OK \\ 73104, USA; Taha-Khan@ouhsc.edu \\ 3 Department Of Family Medicine, University of Oklahoma Health Sciences Center, Oklahoma City, OK \\ 73104, USA; samiya-khan@ouhsc.edu \\ * Correspondence: msiddiq1@numc.edu
}

\begin{abstract}
Chronic eosinophilic pneumonia (CEP) is an idiopathic disorder characterized by an abnormal and marked accumulation of eosinophils in the interstitial and alveolar spaces of the lung. Idiopathic chronic eosinophilic pneumonia is reported to comprise anywhere from $0-2.5 \%$ of cases within the registries of interstitial lung disease. Diagnosis is based on the clinical constellation of symptoms, characteristic radiographic findings and peripheral blood or BAL eosinophilia, in the absence of infection or drug-induced eosinophilia. There is no consensus on the dose and duration of treatment, but most authors recommend initial doses of prednisone at $0.5-1 \mathrm{mg} / \mathrm{kg} / \mathrm{day}$ with gradual tapering of the dose for total treatment duration of 6-12 months.
\end{abstract}

Keywords: eosinophilic pneumonia; lung nodules; dyspnea; eosinophils

\section{INTRODUCTION:}

Chronic eosinophilic pneumonia (CEP) is an idiopathic disorder characterized by an abnormal and marked accumulation of eosinophils in the interstitial and alveolar spaces of the lung. Idiopathic chronic eosinophilic pneumonia is reported to comprise anywhere from $0-2.5 \%$ of cases within the registries of interstitial lung disease.

It is a rare disorder of unknown etiology, and may have a course of sub acute or chronic respiratory symptoms [1]. CEP is predominantly a disease of women and non-smokers, with peak incidence in the fifth and sixth decade of life. Approximately half of these patients have a history of pre-existing asthma or atopic disorders [2].

\section{CASE PRESENTATION:}

A 64 year old African American woman with 40 pack year smoking history was admitted with a two month history of progressively worsening shortness of breath, cough, and significant weight loss of 20lbs. Occasional low grade fever was also reported. Physical examination revealed decreased breath sounds with fine crackles most prominent in the right middle and lower lobes, and mild right upper quadrant pain. Widespread excoriating dermatitis was observed on physical examination, most prominent on the legs bilaterally.

Initial blood work revealed elevated white cell counts with prominent eosinophilia and elevated LDH. Chest x-ray showed multiple bilateral lung nodules. CT scan of the chest, abdomen and pelvis confirmed the findings in the lungs but did not show any widespread disease below the diaphragm. Blood and urine cultures continued to be negative. IgE level was found out to be $>2500$ $\mathrm{IU} / \mathrm{ml}$ with normal values of IgA and IgM. Other diagnostic studies including sputum AFB stain and culture for TB, fungal antigens for histoplasma, coccidiodomycosis and blastomycosis, stool 
ova and parasite examination, Hepatitis panel, HIV, CMV and workup for autoimmune diseases was found to be negative. Broncho-Alveolar Lavage (BAL) revealed 30\% eosinophils in the fluid and biopsy obtained via bronchoscopy confirmed the findings of eosinophilic infilteration.

Treatment was initiated with $0.5 \mathrm{mg} / \mathrm{kg} /$ day of prednisone for chronic eosinophilic pneumonitis. Patient responded very well to the therapy with profound symptomatic relief within the first 72 hours and achieved near complete resolution of symptoms over next 8 weeks.

\section{DISCUSSION:}

CEP was first described by Carrington $\mathrm{CB}$ as a chronic variant of Loffler's syndrome [3]. A rare disorder that affects less than 1 person per 100,000 per year [4].CEP is typically suspected in a patient with progressive dyspnea over one to four months and a chest radiograph showing bilateral peripheral or pleural-based opacities. Severe illness is characterized by high fever, weight loss, night sweats, and shortness of breath. Cough is nearly universal as well as dyspnea, but respiratory failure is rare [4-6]. Non-pulmonary symptoms (aside from fever, fatigue, and weight loss) are uncommon. In CEP eosinophil count is almost always greater than $25 \%$ on BAL. Asthma precedes CEP in half of all cases and peripheral eosinophilia is present at presentation in 80-90\% [4].

Diagnosis is based on the clinical constellation of symptoms, characteristic radiographic findings and peripheral blood or BAL eosinophilia, in the absence of infection or drug-induced eosinophilia. Radiographically opacities are seen which are usually found in an apical location. Opacities surrounding the lung have the appearance of a photographic negative shadow usually seen in pulmonary edema [4]. Characteristic CT findings include areas of bilateral ground-glass opacity distributed peripherally in the middle or lower lungs.

There is broad range of diseases which may present with a similar presentation, differential diagnoses include sarcoidosis, cryptogenic organizing pneumonia, idiopathic hypereosinophilic syndrome, hypersensitivity pneumonitis, and autoimmune respiratory disease (such as non-specific interstitial pneumonia) [4].

There is a dramatic response to steroid therapy and most patients will have subjective improvement within 48 hours and radiographic resolution within 1 week. Fewer than $10 \%$ of patients resolve spontaneously and the symptoms commonly recur with discontinuation within 6 months [1]. Additionally, untreated cases can progress to irreversible

fibrotic changes in the lung [8].There is no consensus on the dose and duration of treatment, but most authors recommend initial doses of prednisone at $0.5-1 \mathrm{mg} / \mathrm{kg} / \mathrm{day}$ with gradual tapering of the dose for total treatment duration of 6-12 months [4-8].

\section{CONCLUSION:}

The relatively nonspecific nature of the clinical presentation of this disease process makes the diagnosis a unique challenge and should be considered when a patient presents with dry cough and dyspnea for over 1 week. Once the diagnosis is established therapy should be initiated with steroids, the response to corticosteroids is so characteristic that rapid improvement to a "therapeutic trial" of steroids in the proper clinical and radiographic context can be diagnostic, thus obviating the need for biopsy. However, but keeping in view the higher relapse rate most patients require long-term treatment and care. 


\section{REFERENCES:}

1. Jederlinic PJ, Sicilian L, Gaensler EA. Chronic eosinophilic pneumonia. A report of 19 cases and a review of the literature. Medicine (Baltimore) 1988;67:154-62

2. Sujith V Cherian, Elena Thampy. 'Photographic negative of pulmonary oedema': a classical radiographic pattern of chronic eosinophilic pneumonia. Postgrad Med J 2015;91:411-412

3. Carrington CB, Addington WW, Goff AM, et al. Chronic eosinophilic pneumonia. N Engl J Med 1969;280:787-98

4. Marchand E, Cordier JF.Idiopathic chronic eosinophilic pneumonia. Orphanet J Rare Dis 2006; 1:11.

5. Gaensler EA, Carrington CB. Peripheral opacities in chronic eosinophilic pneumonia: the photographic negative of pulmonary edema. AJR Am J Roentgenol 1977;128:1-13

6. Matsuse H, Shimoda T, Fukushima C, et al. Diagnostic problems in chronic eosinophilic pneumonia. J Int Med Res. 1997;25:196.

7. Yoshida K, Shijubo N, Koba H, et al. Chronic eosinophilic pneumonia progressing to lung fibrosis. Eur Respir J. 1994;7:1541.

8. Kolb, AG ; Ives, St ; Davies, Sf. Diagnosis in Just Over a Minute: a Case of Chronic Eosinophilic Pneumonia. Journal Of General Internal Medicine, 2013 Jul, 2013;28(7):972-975

(C) 2016 by the authors; licensee Preprints, Basel, Switzerland. This article is an open access article distributed under the terms and conditions of the Creative Commons by Attribution (CC-BY) license (http://creativecommons.org/licenses/by/4.0/). 